Old Dominion University

ODU Digital Commons

Bioelectrics Publications

Frank Reidy Research Center for Bioelectrics

1991

\title{
Electric Field Induced Emission as a Diagnostic Tool for Measurement of Local Electric Field Strengths
}

\author{
A. N. Dharamsi \\ Old Dominion University \\ K. H. Schoenbach \\ Old Dominion University
}

Follow this and additional works at: https://digitalcommons.odu.edu/bioelectrics_pubs

Part of the Electrical and Electronics Commons, Plasma and Beam Physics Commons, and the Quantum Physics Commons

\section{Repository Citation}

Dharamsi, A. N. and Schoenbach, K. H., "Electric Field Induced Emission as a Diagnostic Tool for Measurement of Local Electric Field Strengths" (1991). Bioelectrics Publications. 265.

https://digitalcommons.odu.edu/bioelectrics_pubs/265

\section{Original Publication Citation}

Dharamsi, A. N., \& Schoenbach, K. H. (1991). Electric field induced emission as a diagnostic tool for measurement of local electric field strengths. Journal of Applied Physics, 69(5), 2889-2895. doi:10.1063/1.348598

This Article is brought to you for free and open access by the Frank Reidy Research Center for Bioelectrics at ODU Digital Commons. It has been accepted for inclusion in Bioelectrics Publications by an authorized administrator of ODU Digital Commons. For more information, please contact digitalcommons@odu.edu. 


\title{
Electric field induced emission as a diagnostic tool for measurement of local electric field strengths
}

\author{
A. N. Dharamsi and K. H. Schoenbach \\ Department of Electrical and Computer Engineering and Physical Electronics Research Institute, Old \\ Dominion University, Norfolk, Virginia 23529-0246
}

(Received 17 September 1990; accepted for publication 13 November 1990)

\begin{abstract}
The phenomenon of electric field induced (EFI) emission is examined in several diatomic and polyatomic molecules. The possibility of using this phenomenon as a diagnostic tool to measure, nonintrusively, the strength and direction of local electric fields in plasmas is discussed. An estimate of the EFI signal emitted in a typical application plasma is given. This yields a lower bound on the detector sensitivity necessary to exploit EFI emission in practical applications. It is concluded that, at present, the EFI signal could be measured by some very sensitive infrared detection schemes available. Current progress in infrared detector technology, if maintained, could result in the possibility of utilizing EFI emission on a more routine basis. This would allow measurement of electric fields in plasmas of species that are not suitable candidates for any of the other currently available schemes which measure such fields.
\end{abstract}

\section{INTRODUCTION}

A fundamental understanding of transport phenomena in gaseous discharges and plasmas depends critically upon a knowledge of the temporal and spatial dependence of the local electric field vector $\mathbf{E}$, via the ratio $\mathbf{E} / N$. In practical applications (such as microelectronics, plasma processing, etc.) control over $\mathbf{E} / N$ translates into control over the process in question (for example, the etched profile in the etching process). It has long been recognized that Langmuir probe measurements, besides being intrusive in nature, cannot be utilized in reactive plasmas used in etching and thin film deposition. Further, interpretation of the measurements obtained is usually difficult and requires assumptions, made in probe theories, that are generally invalid under the experimental conditions.

There are several successful techniques for nonperturbative measurements of local $\mathrm{E}$ fields that have been developed in the last few years. Moore, Davis, and Gottscho ${ }^{1}$ used laser-induced fluorescence from rotational levels of $\mathrm{BCl}$, in which parity mixing occurs under the influence of an electric field, to obtain sensitive $(40 \mathrm{~V} / \mathrm{cm})$, nonintrusive, in situ, measurements of the local field with high spatial resolution $\left(\leqslant 10^{-4} \mathrm{~cm}^{3}\right)$. Doughty, Lawler, and Salih ${ }^{2}$ measured discharge electric fields by optogalvanic detection of Rydberg atoms, utilizing the linear Stark effect. Kelleher, Delpech, and Weiner ${ }^{3}$ measured a broadening of a valence autoionization resonance in strontium, which occurs because of an electric field dependent mixing with a broader state of opposite parity. Ganguly and Garscadden ${ }^{4}$ have used Rydberg polarization spectroscopy to measure the direction of the local electric field. More work along these lines has been performed by these and other researchers. $^{5}$

This paper investigates the possibility of using electric field induced (EFI) emission as a tool for nonintrusive measurements of local electric field vectors in discharges and plasmas containing diatomic or polyatomic species which have Raman allowed, but infrared forbidden vibra- tional transitions. We first briefly discuss the phenomenon of EFI spectra in homonuclear diatomic molecules. The connection between EFI spectra and the Raman effect is emphasized and this is used to extend the discussion of EFI in diatomic molecules to polyatomics. The connection to the Raman effect shows that the best candidate vibrational modes would be totally symmetric, Raman active, infrared inactive, vibrational modes of polyatomic molecules. Several polyatomic molecules such as $\mathrm{CCl}_{4}, \mathrm{C}_{2} \mathrm{Cl}_{4}, \mathrm{C}_{2} \mathrm{Br}_{4}$, and $\mathrm{SF}_{6}$ are examined. An estimate for the emitted photon flux in a "typical" plasma is given. It is found that the emitted EFI signal could be measured with the most sensitive infrared detectors currently available. We conclude that the EFI emission scheme could become an important method for nonintrusive measurements of local electric fields in plasmas, if the rate of recent progress in infrared detection technology (for instance, in the development of metal oxide semiconductor photomultipliers ${ }^{6}$ ) is sustained. EFI emission would then offer a method for probing species that are not suitable candidates for any of the other current techniques.

\section{ELECTRIC FIELD INDUCED (EFI) SPECTRA}

The discussion below is carried out using a homonuclear diatomic molecule as an example. An analogous argument applies to any normal vibrational mode of a polyatomic molecule which is Raman active, but infrared inactive.

It is well known that symmetric diatomic molecules, not possessing an electric dipole moment, do not exhibit an infrared vibration-rotation spectrum. However, as Condon $^{7}$ first pointed out, when an external electric field is imposed upon such molecules they become infrared active by virtue of induced dipole moments. This can be explained as a limiting case of the Raman effect.

In the latter, an electromagnetic wave at frequency $\omega$ interacts with a molecule in such a way that the scattered 
light has weak components at frequencies different from the main (Rayleigh) scattered beam whose frequency remains unchanged at $\omega$. The inelastically scattered beam contains frequencies $\omega+\omega_{v}$, where $\omega_{v}$ is the vibrational frequency of the molecule. Such an effect occurs because the electronic polarization of a molecule generally depends on the bond length and is therefore modulated at the vibrational frequency. Since the induced polarization, $p$, is proportional to both the polarizability and the $E$ field of the $e-m$ wave, $p$ exhibits, in addition to the main component at $\omega$, beat frequencies $\omega \pm \omega_{v}$, leading to the observed Raman effect. In the limit that the e- $m$ wave frequency tends to zero, the molecule will emit or absorb light of the vibrational frequency $\omega_{v}$. Hence, infrared absorption and emission spectra are induced, and the EFI effect obeys the Raman selection rules $\Delta v=0, \pm 1 ; \Delta j=0, \pm 2$.

An effect intimately connected to the EFI effect, the collisionally induced dipole moment (CID) effect, in which an induced dipole moment occurs during collisions between homonuclear diatomic molecules, was first observed experimentally by Crawford, Welsh, and Locke ${ }^{8}$ and subsequently investigated by others. ${ }^{9}$ EFI absorption spectra in $\mathrm{H}_{2}$ were first measured by Crawford and Dagg ${ }^{10}$ and subsequently also investigated by Buijs and Gush. ${ }^{11}$ Courtois and Jouve ${ }^{12}$ measured the EFI absorption spectrum of $\mathrm{N}_{2}$. Such spectra were measured in polyatomic molecules a year later by Jones. ${ }^{13}$

The intensity of the EFI spectrum depends on the square of the external field strength, and could, therefore, be used to measure the distribution of the field in an electric discharge containing a molecule such as $\mathrm{N}_{2}, \mathrm{O}_{2}$, or a polyatomic such as $\mathbf{S F}_{6}$. Fluorescence measurements have an advantage over absorption spectral measurements in that the signal, albeit weak, would be compared to an essentially zero background signal. This would be counterbalanced by the need to populate excited vibrational states. However, in a gaseous discharge, vibrational excitation by electron impact can be quite efficient. Some examples, including $\mathrm{N}_{2}$ and $\mathrm{SF}_{6}$, are discussed below.

\section{FIELD-INDUCED INFRARED FLUORESCENCE}

The spontaneous emission lifetime of a transition between two levels, $i$ and $f$, is given by the reciprocal of the Einstein $A$ coefficient, which itself is related to the dipole moment matrix element, $\mu_{\mathrm{fi}}$, coupling the two levels through the following relation ${ }^{14}$

$$
A_{\mathrm{fi}}=\frac{64}{3 c^{3} h} \pi^{4} v^{3} \frac{1}{4 \pi \epsilon_{0}} \mu_{\mathrm{fi}}^{2} .
$$

Here,

$$
\mu_{\mathrm{fi}}=\left\langle\psi_{f}|p| \psi_{i}\right\rangle,
$$

and, in the present context, represents the induced dipole moment

$$
\mu_{\mathrm{fi}}=\epsilon_{0} E\left\langle\psi_{f}|\alpha| \psi_{i}\right\rangle .
$$

The polarizability, $\alpha$, can be expanded in a Taylor series:

$$
\alpha \simeq \alpha_{0}+\left(\frac{\partial \alpha}{\partial Q_{0}}\right) Q
$$

where $\alpha_{0}$ is the polarizability of the molecule at its equilibrium bond length, $(\partial \alpha / \partial Q)_{0}$ is the derivative evaluated at this bond length, and $Q$ is the vibrational normal coordinate.

We now outline the significance of Eq. (4) for the present discussion. As mentioned before, one might view the EFI effect as a limiting case of the Raman effect. ${ }^{7}$ In the usual Raman effect an incident electromagnetic wave with frequency $\omega$ is scattered inelastically by a molecule, resulting in radiation at $\omega \pm \omega_{v} \omega_{v}$ is the frequency of the appropriate molecular vibrational mode. The EFI effect is the Raman effect with $\omega=0 .{ }^{7}$ Now, since only the second term in Eq. (4) leads to the Raman effect (the first term, i.e., $\alpha_{0}$, represents Rayleigh scattering), it follows that for the EFI effect under consideration, only this (second) term need be considered.

The Einstein $A$ coefficient relevant to the EFI effect then becomes

$$
A_{\mathrm{if}}=\frac{64}{3 c^{3} h} \pi^{4} v^{3} \frac{1}{4 \pi \epsilon_{0}} \epsilon_{0}^{2}\left|\left\langle\psi_{f}\left|\left(\frac{\partial \alpha}{\partial Q}\right)_{0} Q\right| \psi_{i}\right\rangle\right|^{2} E^{2} .
$$

The matrix element term, $\left|\left\langle\psi_{f}(\partial \alpha / \partial Q)_{0} Q \mid \psi_{i}\right\rangle\right|^{2}$, has to be ultimately obtained experimentally. For $\mathrm{N}_{2}$, this can be deduced from the experimental work of Courtois and Jouve. ${ }^{12}$ This value $\left(\cong 10^{-25} \mathrm{~cm}^{3}\right)$ leads to

$$
A_{\text {if }} \simeq 3 \times 10^{-22} E^{2} \sec ^{-1} \text {. }
$$

for $\mathrm{N}_{2}$, where $E$ is in volts per meter.

If the density of the excited vibrational state is known, an estimate for the expected fluorescence intensity can be obtained. Hence, if, for simplicity, a steady state condition is assumed, the density of vibrationally excited molecules, $N_{i}$, will be given by

$$
N_{i} \simeq \frac{r}{A_{\text {if }}+K_{l}},
$$

where $r$ is the rate at which excitation occurs (for example by electron impact) and $K_{l}$ is the rate of radiationless deactivation.

If emission from a volume $\Delta V=A \Delta l$ is monitored, then

$$
I_{\mathrm{em}}=h v \Delta l \frac{r}{1+\left(K_{l} / A_{\mathrm{if}}\right)} .
$$

Here, $h v$ is the infrared photon energy corresponding to the vibrational energy level spacing.

The rate of excitation of vibrationally excited molecules, $r$, is a complicated function of the conditions in the discharge (mainly the reduced field strength $-E / N$ ), the molecular species, and the discharge constituents. For simple diatomics such as $\mathrm{N}_{2}, \mathrm{CO}, \mathrm{O}_{2}$, Nighan $^{15}$ has obtained the power transferred, $\eta$, to the vibrational and electronic states as a fraction of the Joule heating term $H$. 
Since $r=(\eta / \Delta E) H(1 / \Delta V) \mathrm{cm}^{-3} \mathrm{sec}^{-1}$, where $H$ is the power deposited in the volume $\Delta V, \Delta E$, the vibrational quantum $(\Delta E=h v)$, and $\eta$ the fractional power transferred to the excited vibrational states, it follows that

$$
I_{\mathrm{em}}=\frac{\eta H}{A} \frac{1}{1+\left(K_{l} / A_{\mathrm{if}}\right)} \frac{\mathrm{W}}{m^{2}} .
$$

In Eq. (9) $\eta, H$, and $A_{\text {if }}$ are all functions of $E$. The factor $(\eta H / A)$ represents that part of the power per unit area in the discharge that results in vibrationally excited molecules. The fraction $1 /\left[1+\left(K_{l} / A_{\text {if }}\right]\right.$ represents the effect of competition between the radiative $\left(A_{\text {if }}\right)$ and nonradiative $\left(K_{l}\right)$ processes that lead to a depletion of these excited states. Clearly, if nonradiative losses were zero $\left(K_{l}=0\right)$, all the power (per unit area- $\eta H / A$ ) deposited in the vibrationally excited species would be emitted. Conversely, if the nonradiative losses are overwhelmingly large in comparison to the rate of spontaneous emission, then no fluorescence signal will be seen. The nonradiative losses, represented by $K_{l}$, will mostly involve degradation of the vibrational energy into heat, via vibrational-translational $(V \rightarrow T)$ conversion. For pure $\mathrm{N}_{2}$, the $V \rightarrow T$ relaxation time, at $1 \mathrm{~atm}$, corresponds to $K_{l} \cong 5 \times 10^{4} \mathrm{sec}^{-1}$; for $\mathrm{O}_{2}$, the corresponding value is about two orders of magnitude smaller. Since the deactivation rate is proportional to the number of collisions per unit time, the value of $K_{l}$ is approximately $50 \mathrm{sec}^{-1}$ (Ref. 16) for the relatively low (approximately 1 Torr) pressures in the discharges being considered here. In this case, then, Eqs. (6) and (9) lead to

$$
I_{\mathrm{em}} \leqslant \frac{(\eta H / A)}{\left(1+10^{23} / E^{2}\right)},
$$

for $\mathrm{N}_{2}$. For $E^{2}<10^{23} V^{2} / \mathrm{m}^{2}$, this becomes

$$
I_{\mathrm{em}}<(\eta H / A) 10^{-23} E^{2} \text {. }
$$

It is important to note that, in practice, the measured emission intensity, $I_{\mathrm{em}}$, would be the result of an electric field viewed over a finite path length. Hence, $E$ in the above expression will be, at best, a line-averaged quantity. The method under discussion could only yield values of $E$ at a given point if $E$ was approximately constant over the line of sight (i.e. if $E$ was constant over $\Delta l$ ). While this may be true in specific instances where the spatial variation of $E$ might exhibit some symmetry, $E$ will not, in general, be constant over $\Delta l$. In the latter case, then, one would actually be measuring an average such as $(1 / \Delta L) \int_{0}^{\Delta L} E^{2} d l$. It should be pointed out, however, that this limitation is not a fundamental one. One could, in principle, extract values of the $E$ fields at specific points by deconvolving results of line-averaged measurements over several different paths.

We note that $(\eta H / A)$ would be some function of $E$, and estimates for this factor could be obtained by a numerical analysis of the Boltzmann equation such as that carried out by Nighan. ${ }^{15} \eta H / A$ is in the range $10^{-2}-1$ $\mathrm{W} / \mathrm{cm}^{2}$, yielding, for $E=1 \mathrm{KV} / \mathrm{cm}, I_{\mathrm{em}}$ between $10^{-13}-10^{-15} \mathrm{~W} / \mathrm{cm}^{2}$. This corresponds to approximately $10^{4}-10^{6}$ photons $\mathrm{cm}^{-2} \mathrm{sec}^{-1}$, at the wavelength corresponding to the $\mathrm{N}_{2}$ fundamental.
The EFI signal will compete with background noise from free-free bremsstrahlung and free-bound recombination radiation. For the conditions in a typical discharge such as those being considered here, recombination radiation will be greater than free-free bremsstrahlung. ${ }^{17} \mathrm{~A}$ rough estimate of the recombination radiation intensity can be made ${ }^{17}$ from

$$
\begin{aligned}
\frac{d E_{\mathrm{fb}}}{d v}= & C N_{e} N_{i+1}\left(\frac{\chi_{H}}{k T_{e}}\right)^{3 / 2}\left(\frac{\chi_{i, n}}{\chi_{H}}\right)^{2}\left(\frac{\eta_{n}}{n}\right) g_{\mathrm{bf}} \\
& \exp \left[\left(\chi_{i, n}-h v\right) / k T_{e}\right],
\end{aligned}
$$

which gives the intensity of emission per cc per second in a unit frequency interval from recombination into the $n$th shell of a hydrogen-like ion of charge $i$. Here, $N_{e}$ and $N_{i+1}$ are the densities of the electrons and ions of charge $(i+1), T_{e}$ is the electron temperature, $\chi_{i, n}$ is the ionization potential from the final state, $\eta_{n}$ is the number of places in the $n$th shell which can be occupied by the captured electron, $g_{b f}$ is the bound-free Gaunt correction factor, and $C=1.7 \times 10^{-40} \mathrm{erg} \mathrm{cm}$.

If one assumes $N_{e} \cong N_{i+1} \cong 10^{8} \mathrm{~cm}^{-3}, k T_{e}=1 \mathrm{eV}$, $\chi_{i, n}=5 \mathrm{eV}, \eta_{n}=5, h v=0.25 \mathrm{eV}$, then one obtains, in the present case, a recombination emission photon flux of $8 \times 10^{3} \mathrm{~cm}^{-2} \mathrm{sec}^{-1}$. Hence, for such plasmas, (10) implies that the EFI signal $\left(10^{4}-10^{6}\right.$ photons $\left.\mathrm{cm}^{-2} \mathrm{sec}^{-1}\right)$ will be easily distinguishable from the background recombination and bremsstrahlung continuum.

We note, further, that Eq. (10) assumes thermodynamic equilibrium and, therefore, may be an overestimate of the recombination radiation intensity. In the cathode fall region, where high electric fields on the order of magnitude assumed in the above estimate exist, the conditions are of a highly nonequilibrium nature. The cathode fall region is expected to be "darker" than predicted in Eq. (10). Hence, it might be possible to measure such fields in plasmas with electron densities greater than the $10^{8} \mathrm{~cm}^{-3}$ assumed above. ${ }^{18}$ Many glow discharge sputtering and plasma etching environments have electron densities on the order of $10^{10} \mathrm{~cm}^{-3}$ (Ref. 18). Here, the recombination continuum intensity will be greater than our estimate above. However, in the cathode fall region this background may still be sufficiently lower than the EFI emission signal. We note in this regard that even if the background emission is comparable to the signal it would still be possible to distinguish between the two by performing intensity measurements over a range centered around the EFI emission wavelength. Such a technique would work because the signal is essentially a line, while the background is broad band.

While the signal photon intensity is low, it could be measured by currently available infrared detectors. Hence, for example, $\mathrm{He} 3$ bolometers, operating at 0.3 degrees $\mathrm{K}$, are capable of measuring photon fluxes of between $10^{6}$ and $10^{7}$ per $\mathrm{cm}^{2}$ per sec. ${ }^{19}$ It is clear, nonetheless, that the technique described in this paper would benefit from progress in the field of infrared detection. Along these lines, one should note that progress in the technology of 
metaloxide-semiconductor photomultipliers (MOSPM), which show great promise, ${ }^{6}$ is especially encouraging.

\section{EFI-INDUCED SPECTRA IN POLYATOMIC MOLECULES}

An alternative way to describe the EFI effect in homonuclear diatomics is to say that vibrations in such molecules are infrared-inactive (i.e., do not exhibit an electric dipole infrared spectrum but are Raman-active, i.e., exhibit such a spectrum in the presence of an external electric field-a static one in the discussions above). It follows that, to extend the above discussion to polyatomic molecules, one should look for normal modes of vibration which are Raman active, ${ }^{20}$ but infrared inactive. Group theoretical arguments ${ }^{21}$ show that such vibrational modes are those species for which the polarizability tensor components $\left(\alpha_{i j} ; i=x, y, z ; j=x, y, z\right)$ form a basis, but for which the electric dipole moment components $\left(\mu_{x}, \mu_{y}, \mu_{z}\right)$ do not. Further, since the polarizability components transform as $x^{2}, y^{2}, z^{2}, x y$, etc., ${ }^{22}$ and the dipole moment components transform as $x, y, z$ the appropriate normal vibrational modes can be found from the character table of the point group to which the molecule belongs.

With the diagnostic application in mind, one wants a normal vibrational mode which shows a large intensity in the Raman effect. Since it is known ${ }^{23}$ that totally symmetric vibrational modes exhibit the strongest Raman lines, we restrict ourselves to a discussion of only such normal modes in this section (although, in principle, this is not necessary). The point groups of interest are, therefore, the $D_{n}, D_{n h}, D_{n d}, C_{n h}, S_{n}$ groups, all the cubic groups $\left(T, T_{h}, T_{d}, O\right.$, and $\left.O_{h}\right)$, and the icosahedral group $I_{h}$ as well as $C_{i}$ and $D_{\infty} h$. [Note all point groups which have an inversion center $\left(C_{i}, C_{2 h}, C_{4 h}, C_{6 h}, D_{2 h}, D_{4 h}, D_{6 h}, D_{8 h}, D_{3 d}\right.$ $D_{5 d}, S_{6}, T_{h}, O_{h}, D_{\infty}$, and $I_{h}$ ) to which the principle of $\mathrm{mu}$ tual exclusion ${ }^{20}$ applies (no infrared active transition may be Raman active and vice versa) form a subset of this list.]

The molecules listed in Table I have totally symmetric vibrational modes, denoted by $\boldsymbol{v}_{1}$, which are forbidden in the ir spectrum but are Raman allowed. From a practical point of view it is desired that there not be any strong infrared allowed fundamental, overtone, or combination lines in the near proximity of the Raman-active $v_{1}$ mode. This means that molecules such as acetylene (in which the ir allowed $v_{3}$ line is very strong and near $v_{1}$ ), methane (in which the ir allowed $v_{3}$ line is near $v_{1}$ ), ethylene (in which the strong ir allowed $v_{9}$ line is near $v_{1}$ ) are not suitable candidates.

Among the promising molecules from the incomplete list given in Table $I$ are $\mathrm{N}_{2}$ and $\mathrm{SF}_{6} . \mathrm{N}_{2}$ has been discussed above in Sec. III. We briefly discuss $\mathbf{S F}_{6}$ now.

This molecule belongs to octahedral point group $O_{h}$, the sulfur atom being at the center of the octahedron and the six fluorine atoms at the corners. The molecule possesses 15 normal modes of vibration, consisting of the totally symmetric "breathing" mode $a_{1 g}$, a doubly degenerate mode $e_{g}$, and four triply degenerate modes $t_{1 u}, t_{1 u}, t_{2 g}$, and

TABLE I. Some molecules with Raman-active, infrared inactive totally symmetric vibrational normal modes.

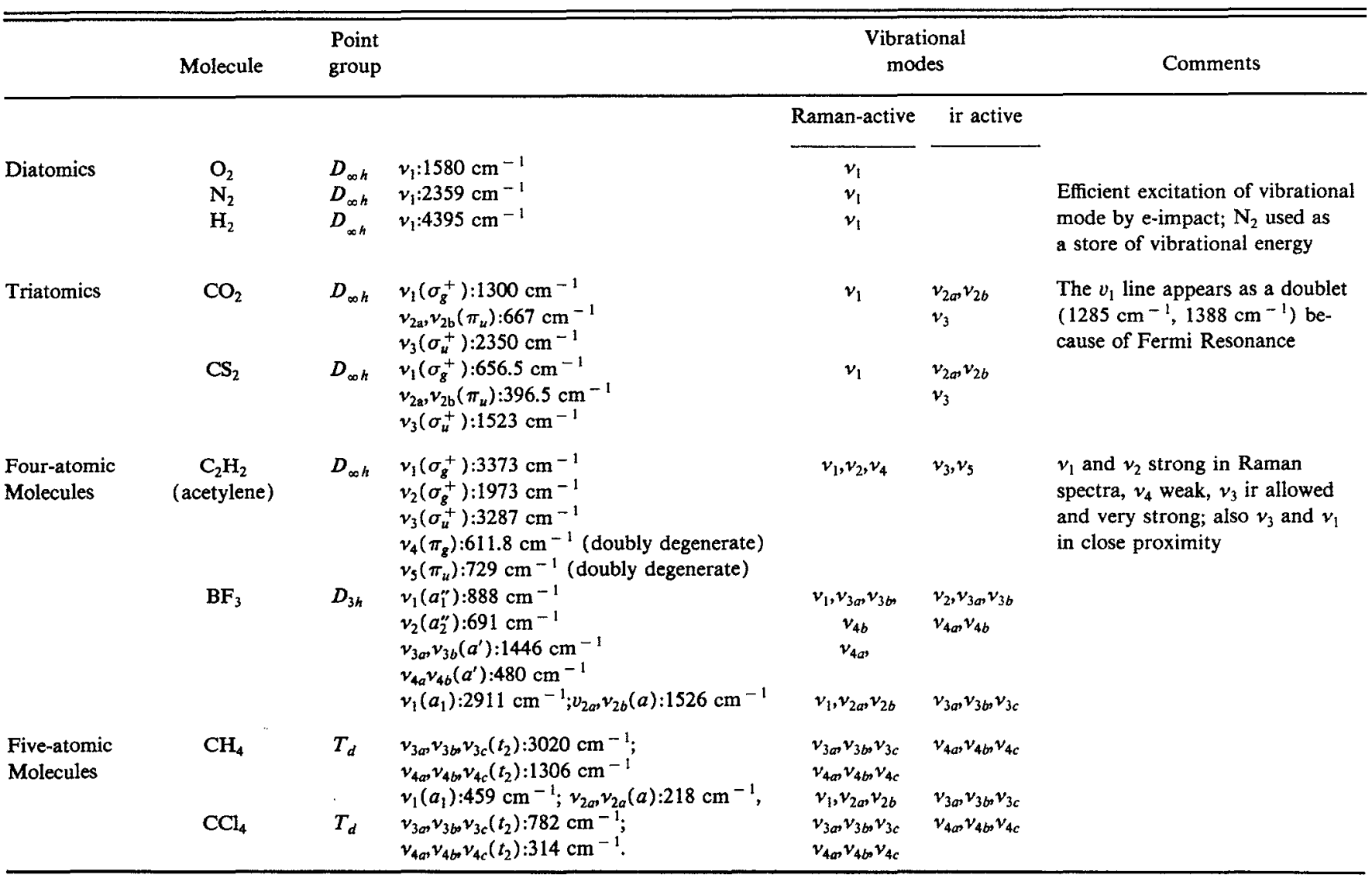


TABLE I. (continued).

\begin{tabular}{|c|c|c|c|c|c|c|c|}
\hline \multirow{3}{*}{$\begin{array}{l}\text { Six-atomic } \\
\text { Molecules }\end{array}$} & \multirow{2}{*}{$\begin{array}{c}\text { Molecule } \\
\mathrm{C}_{4} \mathrm{H}_{2} \\
\text { Diacetylene }\end{array}$} & \multicolumn{2}{|c|}{$\begin{array}{l}\text { Point } \\
\text { group }\end{array}$} & \multicolumn{3}{|c|}{$\begin{array}{l}\text { Vibrational } \\
\text { modes }\end{array}$} & \multirow[t]{2}{*}{ Comments } \\
\hline & & $D_{\infty h h}$ & $\begin{array}{l}v_{1}\left(\sigma_{g}^{+}\right) \\
v_{2}\left(\sigma_{g}^{+}\right) \\
v_{3}\left(\sigma_{g}^{+}\right) \\
v_{4}\left(\sigma_{u}^{+}\right) \\
v_{5}\left(\sigma_{u}^{+}\right) \\
v_{6}\left(\pi_{g}\right) \\
v_{7}\left(\pi_{g}\right) \text { each } \\
v_{8}\left(\pi_{u}\right) \text { doubly } \\
v_{9}\left(\pi_{u}\right) \text { degenerate } \\
{[\text { Ref. } 23, \text { p. } 324]}\end{array}$ & $\begin{array}{c}v_{1}, v_{2} v_{3} \\
v_{6}, v_{7}\end{array}$ & \multicolumn{2}{|c|}{$v_{4}, v_{5}, v_{8}, v_{9}$} & \\
\hline & $\mathrm{C}_{2}$ & $D_{2 h}$ & $\begin{array}{l}v_{1}\left(a_{g}\right): 3019 \mathrm{~cm}^{-1} ; v_{2}\left(a_{g}\right): 1623 \mathrm{~cm}^{-1} \\
v_{3}\left(a_{g}\right): 1342 \mathrm{~cm}^{-1} ; v_{4}\left(a_{u}\right): 825 \mathrm{~cm}^{-1} \\
v_{5}\left(b_{1 g}\right): 3272 \mathrm{~cm}^{-1} ; v_{6}\left(b_{l g}\right): 1050 \mathrm{~cm}^{-1} \\
v_{7}\left(b_{1 u}\right): 949 \mathrm{~cm}^{-1} ; v_{8}\left(b_{2 g}\right): 943 \mathrm{~cm}^{-1} \\
v_{9}\left(b_{2 a}\right): 3105 \mathrm{~cm}^{-1} ; v_{10}\left(b_{2 u}\right): 995 \mathrm{~cm}^{-1} \\
v_{11}\left(b_{3 u}\right): 2989 \mathrm{~cm}^{-1} ; v_{12}\left(b_{3 u}\right): 1443 \mathrm{~cm}^{-1}\end{array}$ & $\begin{array}{l}v_{1}, v_{2}, v_{3} \\
v_{5}, v_{6}, v_{8}\end{array}$ & \multicolumn{2}{|c|}{$\begin{array}{l}v_{7}, v_{9}, v_{10}, v_{11}, \\
v_{12}\end{array}$} & $\begin{array}{l}v_{4} \text { is inactive, } v_{1}, v_{2}, v_{3}, \\
\text { the totally symmetric modes are } \\
\text { very strong Raman lines. There is } \\
\text { a strong ir line ( } v_{9} \text { near } v_{1} \text {; and } \\
\text { another ir strong line }\left(v_{12}\right) \text { near } v_{2} \\
\text { and } v_{3} \text {. This makes the molecule } \\
\text { an unsuitable candidate. }\end{array}$ \\
\hline & $\begin{array}{l}\mathrm{C}_{2} \mathrm{Cl}_{4} \\
\text { Tetrachloroethylene }\end{array}$ & $D_{2 h}$ & $\begin{array}{l}v_{1}\left(a_{g}\right): 1571 \mathrm{~cm}^{-1} ; v_{2}\left(a_{g}\right): 447 \mathrm{~cm}^{-1} \\
v_{3}\left(a_{g}\right): 237 \mathrm{~cm}^{-1} ; v_{4}\left(a_{u}\right): \\
v_{5}\left(b_{1 g}\right): 1000 \mathrm{~cm}^{-1} ; v_{6}\left(b_{1 g}\right): 337 \mathrm{~cm}^{-1} \\
v_{7}\left(b_{1 u}\right): 332 \mathrm{~cm}^{-1} ; v_{8}\left(b_{2 g}\right): 512 \mathrm{~cm}^{-1} \\
v_{9}\left(b_{2 u}\right): 909 \mathrm{~cm}^{-1} ; v_{10}\left(b_{2 u}\right): 213 \mathrm{~cm}^{-1} \\
v_{11}\left(b_{3 u}\right): 782 \mathrm{~cm}^{-1} ; v_{12}: 418 \mathrm{~cm}^{-1}\end{array}$ & Ditto & Ditto & & $\begin{array}{l}\text { EFI induced absorption spectrum } \\
\text { measured experimentally } \\
\text { by Jones. }\end{array}$ \\
\hline & $\begin{array}{c}\mathrm{C}_{2} \mathrm{Br}_{4} \\
\text { Tetrabromoethylene }\end{array}$ & $D_{2 h}$ & Species as in $\mathrm{C}_{2} \mathrm{H}_{4}$ and $\mathrm{C}_{2} \mathrm{Cl}_{4}$ & Ditto & Ditto & & Ditto \\
\hline & $\begin{array}{l}\text { Diphenyl } \\
\text { acetylene }\end{array}$ & $D_{2 h}$ & Ditto & Ditto & Ditto & & Ditto \\
\hline $\begin{array}{l}\text { Seven-atomic } \\
\text { Molecules }\end{array}$ & $\mathrm{SF}_{6}$ & $\begin{array}{l}v_{1}(a \\
v_{2 a}, v \\
v_{3 a}, v \\
v_{4 a}, v \\
v_{5 a}, v \\
v_{6 a}, v\end{array}$ & $\begin{array}{l}g): 775 \mathrm{~cm}^{-1}(12.9 \mu) \\
{ }_{b}\left(l_{g}\right): 644 \mathrm{~cm}^{-1}(15.5 \mu) \\
b_{b r} v_{3 c}\left(t_{1 u}\right): 965 \mathrm{~cm}^{-1}(10.3 \mu) \\
t_{b r} v_{4 c}\left(t_{1 u}\right): 617 \mathrm{~cm}^{-1}(16.2 \mu) \\
b_{b r} v_{5 c}\left(t_{2 g}\right): 524 \mathrm{~cm}^{-1}(19.1 \mu) \\
b_{b}, v_{6 c}\left(t_{2 u}\right): 363 \mathrm{~cm}^{-1}(27.5 \mu)\end{array}$ & & $\begin{array}{l}3 b, v_{3 c} \\
4 b, v_{4 c}\end{array}$ & $\begin{array}{l}v_{60}, v \\
\text { symr } \\
\text { line } \\
\text { and } \\
\text { the s } \\
v_{3}, v_{4} \\
\text { stron } \\
v_{1} \text { an } \\
\text { effici }\end{array}$ & $\begin{array}{l}66, v_{6 c} \text { are inactive. The totally } \\
\text { metric mode } v_{1} \text { appears as a strong } \\
\text { in the Raman spectrum } \\
\text { is sufficiently far removed from } \\
\text { strong ir active modes } \\
\text { There are no } \\
\text { Th overtones or combinations near } \\
\text { nd this mode (as well as } v_{3} \text { ) is } \\
\text { lently excited by electron impact. }\end{array}$ \\
\hline
\end{tabular}

$t_{2 u}{ }^{23}$ The fundamentals of these species are designated as $v_{1}, v_{2}, v_{3}, v_{4}, v_{5}$, and $v_{6}$, respectively. The $v_{1}, v_{2}$, and $v_{5}$ fundamentals are forbidden by the electric dipole selection rule, however they are all Raman active. The $v_{3}$ and $v_{4}$ modes are ir active but Raman inactive. The final fundamental, $v_{6}$, is neither infrared nor Raman active.

Being totally symmetric, the $v_{1}$ mode appears as a very strong line in the Raman spectrum. ${ }^{23}$ Also, there are no strong infrared fundamentals, overtones, or combinations near this frequency. This fundamental can therefore be expected to appear as an EFI line and would be fairly distinct.

The $v_{1}$ mode would be a suitable candidate for monitoring EFI fluorescence in a discharge because it is one of the two modes that are efficiently excited by electron impact. The other mode is the strongly infrared-active $v_{3}$ mode. Rohr's ${ }^{24,25}$ results indicate that the integrated total vibrational excitation is about $10^{-15} \mathrm{~cm}^{-2}$, being about 1.5 times the elastic cross section. The vibrational excitation cross section is dominated by the $v_{1}$ and $v_{3}$ modes, with some excitation of the infrared active $v_{4}$ mode also occurring. The $v_{3}(10.3 \mu)$ and $v_{4}(16.2 \mu)$ fundamentals are sufficiently removed from $v_{1}(12.9 \mu)$ so as not to interfere with any EFI fluorescence. As discussed above, the noise at $12.9 \mu$ that would have to be overcome would be principally from recombination radiation.

\section{DISCUSSION}

A possible experimental arrangement is shown below in Fig. 1. An electron beam would produce vibrationally excited molecules. The sample chamber would hold the molecule under investigation, in a rare gas buffer. Infrared emission (at the fundamental lines given in Table I) would be monitored, in the presence and absence of an electric field.

The EFI emission intensity is given by

$$
I_{\mathrm{em}}=K \Delta l / h v N_{i} E^{2}
$$




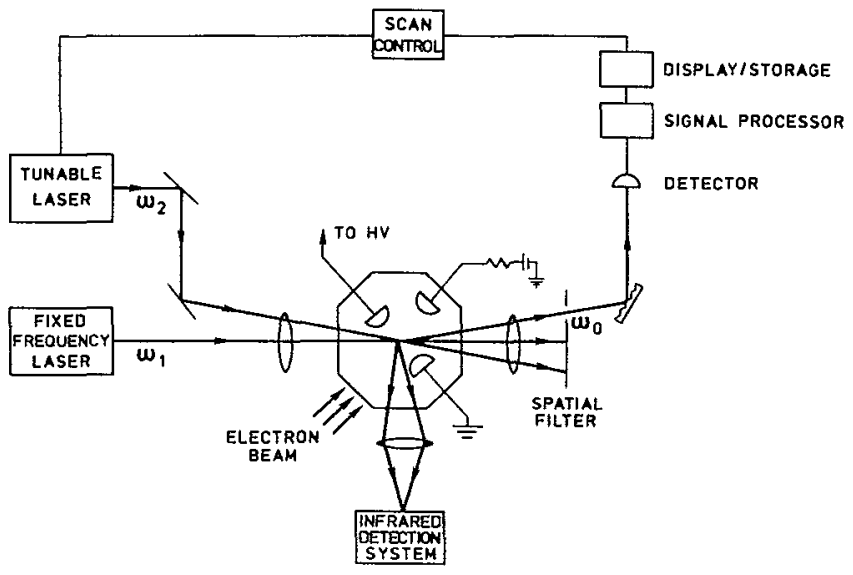

FIG. 1. Proposed experimental arrangement.

where $K$ is the factor on the right-hand side of Eq. (5) which multiples $E^{2}, \Delta l$ the path length being monitored, and $N_{i}$ the density of excited vibrational levels produced. $K$ depends on the molecular transition being monitored through the induced dipole moment matrix element. The vibrationally excited state density, $n_{i}$, could be measured by Coherent Antistokes Raman Spectroscopy (CARS).

From the viewpoint of a diagnostic application, we note that Eq. (11) yields

$$
E_{1}=E_{0}\left(\frac{I_{\mathrm{em}_{1}}}{I_{\mathrm{em}_{0}}}\right)^{1 / 2}\left(\frac{\Delta l_{0}}{\Delta l_{1}}\right)^{1 / 2}\left(\frac{N_{i 0}}{N_{i 1}}\right)^{1 / 2},
$$

where the subscript 1 denotes values relevant to the condition in which the local electric field is to be measured, and the subscript 0 denotes values that would be obtained in a calibration experiment to be conducted in an apparatus such as that shown in Fig. 1. In the calibration experiment $E_{0}$ would be known because the experiment would be conducted with a high impedance in series with the electrodes so as to prevent a discharge. Equation (12) shows that, then, it is necessary to obtain only the ratios of the measured intensities and excited state population densities in order to find the local electric field strength to be measured, $E_{1}$. A measurement of such a ratio could be obtained by a CARS type arrangement, such as that depicted in Fig. 1. The latter is an adaptation of a standard CARS setup (see, for example, Ref. 26).

The EFI effect can be viewed as a limiting case (with the frequency of incident radiation equal to zero) of Raman scattering and, hence, obeys the Raman selection rules. EFI absorption and the process leading to the Stokes Raman line are analogous, because in both cases, a molecule initially in the ground state makes a transition to an excited vibration-rotation level after interacting with an electromagnetic field. On the other hand, EFI emission and the antistokes Raman process are analogous because in both these cases a molecule, initially in an excited vibration-rotation level makes a transition to a lower level, under the influence of an electromagnetic field and contributes a quantum of energy to the latter. The close analogy

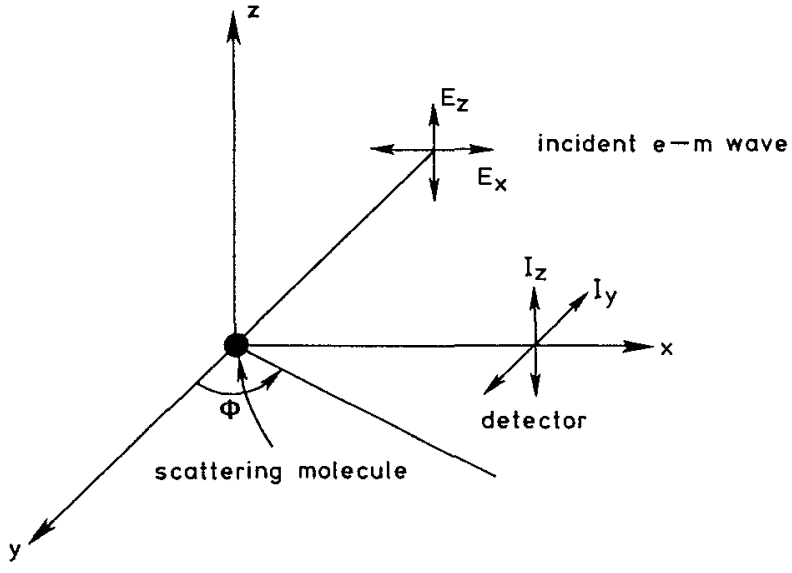

FIG. 2. Depolarization measurements in Raman scattering.

between the EFI and Raman effects means that, in principle, the direction of the electric field could be determined by measurement of depolarization ratios.

In the Raman effect (see Fig. 2) when the incident beam is polarized in a direction perpendicular to the direction of scattering (i.e., $E_{x}=0$ ), the depolarization ratio is given by

$$
\rho_{P \perp}=\frac{I_{y}}{I_{z}}=\frac{6 \gamma_{\mathrm{nm}}^{2}}{45\left(\alpha_{\mathrm{nm}}\right)^{2}+4 \gamma_{\mathrm{nm}}^{2}},
$$

where $\alpha_{\mathrm{nm}}$ and $\gamma_{\mathrm{nm}}$ are, respectively, the matrix elements of the mean value and anisotropy invariants of polarizability. Equation (13) also applies to emission induced by a static electric field, pointing along the $z$ axis, as in Fig. 2. In this case, because of symmetry, the depolarization ratio would be independent of the azimuthal angle $\phi$. This would provide a means for determining the direction of the electric field. For instance, the measurement of a constant depolarization around $2 \pi$ radians in a given circle centered on the scatterer indicates that the $E$ field is perpendicular to this plane. (Once again, as mentioned in Sec. III, this discussion regarding extraction of information about the field direction from emission polarization measurements must be tempered with the realization that, in practice, the emission detected is an average over the line of sight, $\Delta l$. Hence, this simplified discussion applies, strictly speaking, to only those lines of light over which the $E$ field vector, i.e., its magnitude as well as direction are constant.)

The technique of EFI fiuorescence could, in principle, be extended to measurements in the liquid as well as solid phases. For example, the fundamental optical lattice vibrational mode in crystals of octahedral symmetry $O_{h}$ such as diamond, silicon, and germanium are infrared inactive but Raman allowed (cf. $\mathrm{SF}_{6}$ in Table I). (Weak absorption bands, explained by the existence of higher order electric moments have been observed ${ }^{27}$.) It might be possible to observe optical phonon transitions which become allowed in the presence of $E$ fields and hence obtain a measurement of the latter.

In concluding, we note that an electric field intensity of $1 \mathrm{kV} / \mathrm{cm}$ (a value that might be expected in the cathode fall region of a discharge) would produce an EFI emission 
photon flux of up to $10^{6}$ photons $/ \mathrm{cm}^{2} / \mathrm{sec}$. This is a level that can be detected by the sensitive infrared detectors currently available (for example the $\mathrm{He} 3$ bolometer system). We note also that if sufficient progress occurs in infrared detector technology, EFI emission would be an attractive method for measuring local fields in plasmas of species that are not suitable candidates in other currently available diagnostic techniques.

\section{ACKNOWLEDGMENTS}

It is a pleasure to acknowledge helpful discussions with Dr. A. Garscadden, Dr. B. N. Ganguly, and Dr. Glenn Gerdin.

'C. A. Moore, G. P. Davis, and R. A. Gottscho, Phys. Rev. Lett. 52, 538 (1984).

${ }^{2}$ D. K. Daughty and J. E. Lawler, Appl. Phys. Lett. 45, 611 (1984); D. K. Daughty, S. Salih, and J. E. Lawler, Phys. Lett. 103A, 41 (1984).

${ }^{3}$ D. E. Kelleher, J. F. Delpech, and J. Weiner, Phys. Rev. A 32, 2230, 1985.

${ }^{4}$ B. N. Ganguly and A. Garscadden, ibid. paper KB-3.

${ }^{5}$ B. N. Ganguly, J. Shoemaker, B. L. Preppernau, A. Garscadden, and R. A. Gottscho, 39th Annual Gaseous Electronics Conference, paper KB-2, Madison, Wisconsin, 1986; R. A. Gottscho, G. R. Scheller, and David Stoneback, J. Appl. Phys. 66, 492 (1989); T. J. Sommerer, W. N. G. Hitchon, and J. E. Lawler, Phys. Rev. A 39, 6356 (1989).

${ }^{6}$ L. F. Lou and G. L. Tettmer, J. Appl. Phys, 66, 2678 (1989).

${ }^{7}$ E. U. Condon, Phys. Rev. 41, 759 (1932).
${ }^{8}$ M. F. Crawford, H. L. Welsh, and J. L. Locke, Phys. Rev. 75, 1607 (1949).

${ }^{9}$ Z. J. Kiss and H. L. Welsh, Can. J. Phys. 37, 1249 (1959); J. Van Kranendonk, Physica 23, 825 (1957); and 24, 347, 1958; G. Varghese and S. Paddi Reddy, Can. J. Phys. 47, 2745 (1969); A. K. Kudian and H. L. Welsh, Can. J. Phys. 47, 2745 (1969).

${ }^{10}$ M. F. Crawford and I. R. Dagg, Phys. Rev. 91, 1569 (1953).

${ }^{11}$ H. L. Buijs and H. P. Gush, Can. J. Phys. 49, 2366 (1971).

${ }^{12}$ D. Courtois and P. Jouve, J. Mol. Spectrosc. 55, 18 (1975).

${ }^{13}$ D. E. H. Jones, J. Chem. Soc. Faraday Trans. 72, 1397 (1976).

${ }^{14}$ R. C. Tolman, Phys. Rev. 23, 693 (1924).

${ }^{15}$ W. L. Nighan, Phys. Rev. 2, 1989 (1970).

${ }^{16}$ A. B. Callear and J. D. Lambert, in Comprehensive Chemical Kinetics, edited by C. H. Bamford and C. F. H. Tipper (Elsevier, New York, 1969), Vol. 3.

${ }^{17}$ T. F. Stratton, in Plasma Diagnostic Techniques, edited by R. H. Huddlestone and S. L. Leonard (Academic, New York, 1965).

${ }^{18}$ B. Chapman, Glow Discharge Processes; Sputtering and Plasma Etching (Wiley, New York, 1980).

${ }^{19}$ Private communication, Infrared Laboratories, Tucson, Arizona.

${ }^{20}$ L. A. Woodward, Nature 165, 198 (1950).

${ }^{2 !}$ F. A. Cotton, Chemical Applications of Group Theory (Wiley, New York, 1971).

${ }^{22}$ E. B. Wilson, J. C. Decius, and P. C. Cross, Molecular Vibrations (McGraw-Hill, New York, 1955).

${ }^{23}$ G. Herzberg, Infrared and Raman Spectra (Van Nostrand, New York, 1945); L. A. Woodward, Introduction to the Theory of Molecular Vibrations and Vibrational Spectroscopy (Oxford, London, 1972).

${ }^{24}$ K. Rohr, J. Phys. B 10, 1175 (1977).

${ }^{25}$ K. Rohr, J. Phys. B 12, L185 (1979).

${ }^{26} \mathrm{James}$ J. Valentini, in Fast Electrical and Optical Measurements, edited by J. E. Thompson and L. H. Luessen, NATO ASI Series E, No. 109 (Martinus Nijhoff, Boston, 1986), Vol. II.

${ }^{27}$ M. Lax and E. Burstein, Phys. Rev. 97, 39 (1955). 